\title{
EULAR/ERA-EDTA recommendations for the management of ANCA-associated vasculitis
}

\author{
M Yates, ${ }^{1,2}$ R A Watts, ${ }^{2,3}$ I M Bajema, ${ }^{4}$ M C Cid, ${ }^{5}$ B Crestani, ${ }^{6}$ T Hauser $^{7}$ \\ B Hellmich, ${ }^{8} \mathrm{~J}$ U Holle, ${ }^{9} \mathrm{M}$ Laudien, ${ }^{10} \mathrm{M}$ A Little, ${ }^{11} \mathrm{R}$ A Luqmani, ${ }^{12} \mathrm{~A}$ Mahr, ${ }^{13}$ \\ P A Merkel, ${ }^{14}$ J Mills, ${ }^{15}$ J Mooney, ${ }^{1}$ M Segelmark, ${ }^{16,17} \mathrm{~V} \mathrm{Tesar}^{18}{ }^{18} \mathrm{~K}$ Westman, ${ }^{19}$ \\ A Vaglio, ${ }^{20} \mathrm{~N}$ Yalçındağ, ${ }^{21}$ D R Jayne, ${ }^{22}$ C Mukhtyar $^{1}$
}

\begin{abstract}
Handling editor Tore K Kvien
- Additional material is published online only. To view please visit the journal online (http://dx.doi.org/10.1136/ annrheumdis-2016-209133).

For numbered affiliations see end of article.

\section{Correspondence to} Dr Chetan Mukhtyar, Department of Rheumatology, Norfolk and Norwich University Hospital, Norwich NR4 7UY UK; chetan.mukhtyar@nnuh. nhs.uk
\end{abstract}

Received 5 January 2016 Revised 24 May 2016 Accepted 27 May 2016 Published Online First 23 June 2016

\section{CrossMark}

\footnotetext{
To cite: Yates $M$, Watts RA, Bajema IM, et al. Ann Rheum Dis 2016;75: 1583-1594.
}

\section{ABSTRACT}

In this article, the 2009 European League Against Rheumatism (EULAR) recommendations for the management of antineutrophil cytoplasmic antibody (ANCA)-associated vasculitis (AAV) have been updated. The 2009 recommendations were on the management of primary small and medium vessel vasculitis. The 2015 update has been developed by an international task force representing EULAR, the European Renal Association and the European Vasculitis Society (EUVAS). The recommendations are based upon evidence from systematic literature reviews, as well as expert opinion where appropriate. The evidence presented was discussed and summarised by the experts in the course of a consensus-finding and voting process. Levels of evidence and grades of recommendations were derived and levels of agreement (strengths of recommendations) determined. In addition to the voting by the task force members, the relevance of the recommendations was assessed by an online voting survey among members of EUVAS. Fifteen recommendations were developed, covering general aspects, such as attaining remission and the need for shared decision making between clinicians and patients. More specific items relate to starting immunosuppressive therapy in combination with glucocorticoids to induce remission, followed by a period of remission maintenance; for remission induction in lifethreatening or organ-threatening $A A V$, cyclophosphamide and rituximab are considered to have similar efficacy; plasma exchange which is recommended, where licensed, in the setting of rapidly progressive renal failure or severe diffuse pulmonary haemorrhage. These recommendations are intended for use by healthcare professionals, doctors in specialist training, medical students, pharmaceutical industries and drug regulatory organisations.

\section{INTRODUCTION}

Granulomatosis with polyangiitis (GPA, Wegener's granulomatosis), microscopic polyangiitis (MPA) and eosinophilic granulomatosis with polyangiitis (EGPA, Churg-Strauss syndrome) are termed the antineutrophil cytoplasmic antibody (ANCA)associated vasculitides (AAVs). ${ }^{1}$ GPA, MPA and EGPA have respective annual incidence rates of 2.1-14.4, 2.4-10.1 and $0.5-3.7$ per million in Europe, and the prevalence of AAV is estimated at to be $46-184$ per million. ${ }^{2-8}$ The 5 -year survival rates for GPA, MPA and EGPA are estimated to be 74-91\%, 45-76\% and 60-97\%, respectively. ${ }^{9}$

\section{BACKGROUND AND RATIONALE}

In 2009 the European League Against Rheumatism (EULAR) published recommendations for managing primary small and medium vessel vasculitis which included the management of $\mathrm{AAV}^{10}$ The publication of 1691 papers in the past 5 years on primary systemic vasculitis in internal medicine, rheumatology and nephrology journals, as well as the licensing of rituximab for $\mathrm{AAV}$, make this an opportune time to update the recommendations with an AAV focus. This update was made in conjunction with the European Renal Association-European Dialysis and Transplant Association (ERA-EDTA).

This paper reassesses standard therapy, including the use of biological agents, the prognostic value of histopathology and management of long-term complications, integrating these into treatment algorithms.

\section{METHODS}

The EULAR standardised operating procedure for the elaboration, evaluation, dissemination and implementation of recommendations were followed. ${ }^{11}$ The full details are available in the online supplementary material. The task force comprised 21 members representing EULAR and ERA-EDTA: a patient (John Mills), a nurse (Janice Mooney), a pathologist (IMB), an otorhinolaryngologist (ML), a pulmonologist (BC), an immunologist (TH), an ophthalmologist (NY), two general internists (AM, MCC), six renal physicians (MAL, MS, VT, KW, AV and DRJ) and six rheumatologists (RAW, BH, JUH, RAL, PAM and CM) with academic experience and/or clinical expertise in the field of vasculitis. MY was the Clinical Fellow.

A Delphi exercise was conducted to identify items needing update and new items. This instructed the systematic literature review (SLR) strategy. The manuscripts were formally scored using the Critical Appraisal Skills Programme checklist (http://www. casp-uk.net/). Details are available in the online supplement.

\section{STATEMENTS}

The statements in this manuscript are termed 'recommendations' as opposed to 'guidelines' or 'points to consider' because they offer guidance which needs to be tailored to meet individual requirements (table 1). They are intended for use by healthcare professionals, doctors in specialist training, medical students, pharmaceutical industries and drug regulatory organisations. An algorithm has been developed to reflect the statements (figure 1). 


\section{Table 1 Recommendation statements}

\section{Statement}

1. We recommend that patients with AAV are managed in close collaboration with, or at, centres of expertise.

2. A positive biopsy is strongly supportive of a diagnosis of vasculitis and we recommend biopsies to assist in establishing a new diagnosis and for further evaluation for patients suspected of having relapsing vasculitis.

3. For remission-induction of new-onset organ-threatening or life-threatening AAV we recommend treatment with a combination of glucocorticoids and either cyclophosphamide OR rituximab.

4. For remission-induction of non-organ-threatening AAV we recommend treatment with a combination of glucocorticoids and either methotrexate or mycophenolate mofetil*.

5. For a major relapse of organ-threatening or life-threatening disease in AAV we recommend treatment as per new disease with a combination of glucocorticoids and either cyclophosphamide OR rituximab.

6. (i) Plasma exchange should be considered for patients with $A A V$ and a serum creatine level of $\geq 500 \mu \mathrm{mol} / \mathrm{L}$ $(5.7 \mathrm{mg} / \mathrm{dL}$ ) due to rapidly progressive glomerulonephritis in the setting of new or relapsing disease.

6. (ii) Plasma exchange can also be considered for the treatment of severe diffuse alveolar haemorrhage.

7. For remission-maintenance of AAV we recommend treatment with a combination of low-dose glucocorticoids and either azathioprine, rituximab, methotrexate or mycophenolate mofetil*.

8. We recommend that remission-maintenance therapy for AAV be continued for at least 24 months following induction of sustained remission.

9. For patients with AAV refractory to remission-induction therapy we recommend switching from cyclophosphamide 3 to rituximab or from rituximab to cyclophosphamide. These patients should be managed in close conjunction with, or referred to, an expert centre for further evaluation and potential enrolment in clinical trials.

10. We recommend that structured clinical assessment rather than ANCA testing should inform decisions on changes 4 in treatment for AAV.

11. We recommend the investigation of persistent unexplained haematuria in patients with prior exposure to cyclophosphamide.

12. Hypoimmunoglobulinaemia has been noted after treatment with rituximab. We recommend testing of serum immunoglobulin levels prior to each course of rituximab and in patients with recurrent infection.

13. We recommend periodic assessment of cardiovascular risk for patients with AAV.

14. We recommend that patients with $\mathrm{AAV}$ should be given a clear verbal explanation of the nature of their disease, 3 the treatment options, the side effects of treatment, and the short-term and long-term prognoses.

15. We recommend that following the remission-induction phase of treatment, patients with AAV be assessed for the 4 extent and ongoing impact of comorbidities associated with their diagnosis. Patients should then be advised where they might find the necessary therapies or support for these conditions.

*The drugs are listed in order of the strength of vote (see text).

AAV, ANCA-associated vasculities; ANCA, antineutrophil cytoplasmic antibody; AZA, azathioprine; CYC, cyclophosphamide; EGPA, eosinophilic granulomatosis with polyangiitis; GPA, granulomatosis with polyangiitis; MMF, mycophenolate mofetil; MPA, microscopic polyangiitis; MTX, methotrexate; RTX, rituximab.

AAV is a very variable disease group which is unpredictable and potentially life-threatening. Treatment usually involves potent immunosuppressive drugs, often with risk of significant side effects. Full drug-free remission can be achieved but relapse is common. In addition, AAV adversely affects quality of life even in patients thought to have clinical remission. ${ }^{12} 13$ This may be an effect of the disease or its treatment. We recommend the overarching principle of shared decision making between the patient and their specialist.

\section{Statement 1}

We recommend that patients with AAV are managed in close collaboration with, or at, centres of expertise. Level of evidence 3; grade of recommendation C; strength of vote $100 \%$.

The rarity of AAV makes it difficult to maintain expertise in their management. ${ }^{2}{ }^{14-16}$ Assessment of these patients requires expert guidance to differentiate activity from damage or infection and to consider differential diagnoses. Patients may require interventions by specialists with expertise in AAV, such as immunological monitoring, use of rituximab in patients with refractory disease, specialised radiography, assessment of eye involvement, injection of subglottic stenosis and renal transplantation. ${ }^{17-24}$ For patients with refractory disease, the best option may be consideration of referral to centres participating in clinical trials. AAV may relapse years after remission is achieved, even in previously unaffected organ systems. ${ }^{25-27}$ Patients may develop complications from the treatment many years after discontinuing treatment. ${ }^{28}{ }^{29}$ Long-term follow-up and rapid access to specialist services are necessary for all patients with AAV. For these reasons patients with AAV should be managed in close collaboration with, or at, centres of expertise. ${ }^{30}$

\section{Statement 2}

A positive biopsy is strongly supportive of a diagnosis of vasculitis and we recommend biopsies to assist in establishing a new diagnosis and for further evaluation for patients suspected of having relapsing vasculitis. Level of evidence 3; grade of recommendation C; strength of vote $81 \%$.

A positive biopsy for AAV is helpful when considering an initial diagnosis or recurrent disease. Histopathological evidence of vasculitis, such as pauci-immune glomerulonephritis or necrotising vasculitis in any organ, remains the gold standard for diagnostic purposes. The likely diagnostic yield varies and is dependent on the organ targeted. In patients with GPA with renal involvement the diagnostic yield from renal biopsy can be as high as $91.5 \%{ }^{31}$ Otorhinolaryngological examination in patients with GPA often reveals abnormal findings and biopsies of these areas may be positive for inflammatory changes in up to $68.4 \% .{ }^{32}{ }^{33} \mathrm{~A}$ large study of 60 nasal, 27 paranasal sinus, 17 laryngeal, 5 periorbital, 5 oral, 4 middle ear, 3 mastoid, 2 external ear and 3 salivary gland 


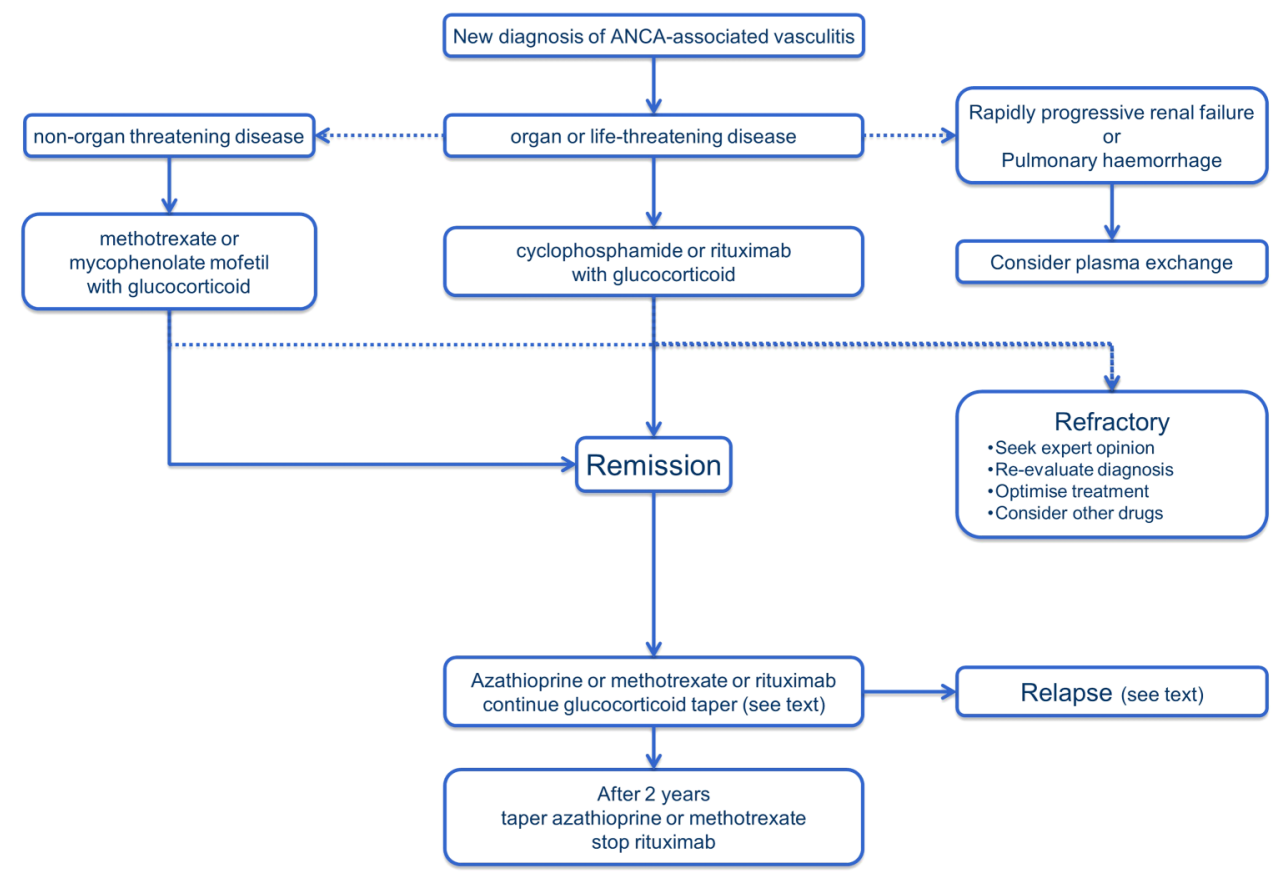

Figure 1 Algorithm to describe the management of new antineutrophil cytoplasmic antibody (ANCA)-associated vasculitis. Dashed lines indicate alternative or supplementary action to consider.

biopsies revealed that they often yield non-specific chronic inflammation and the more specific findings of granulomas and vasculitis are seen less frequently than in other tissue biopsies. ${ }^{34}$ Lung biopsies vary in their diagnostic sensitivity, with only $12 \%$ of transbronchial biopsies of alveolar tissue positive for GPA and 66.7\% for EGPA in one study. ${ }^{32}$ Open lung biopsies, although more invasive, provide a much higher diagnostic yield. ${ }^{35}$

Percutaneous renal biopsy should be performed using ultrasound guidance where possible and has been shown to be associated with a low risk of complications including haemorrhage. $^{36}$ The risk of bleeding following percutaneous renal biopsy is higher in patients treated with plasma exchange (PLEX). ${ }^{37}$ Generic factors associated with an increased risk of bleeding necessitating transfusion include old age, increased systolic blood pressure and worse renal function. ${ }^{38}$

Existing classification systems need further validation but changes like glomerular sclerosis have obvious adverse prognostic value for patients with $\mathrm{AAV}^{39-41}$

\section{Statement 3}

For remission-induction of new-onset organ-threatening or lifethreatening AAV we recommend treatment with a combination of glucocorticoids and either cyclophosphamide OR rituximab.

- Cyclophosphamide

- level of evidence 1A for GPA and MPA; grade of recommendation A; strength of vote $100 \%$.

- level of evidence 3 for EGPA; grade of recommendation C; strength of vote $88 \%$.

- Rituximab

- level of evidence 1B for GPA and MPA; grade of recommendation A; strength of vote $82 \%$.

- level of evidence 3 for EGPA; grade of recommendation C; strength of vote $59 \%$.

Since the 1970 s therapy consisting of a combination of glucocorticoids ( $1 \mathrm{mg} / \mathrm{kg} /$ day-maximum daily dose $80 \mathrm{mg}$ ) with cyclophosphamide ( $2 \mathrm{mg} / \mathrm{kg} /$ day-maximum $200 \mathrm{mg} /$ day $)$ has been used for remission induction in $\mathrm{AAV}^{42}$ Due to concerns about cumulative cyclophosphamide dosage, pulsed intravenous regimens were designed and tested, the largest study being the CYCLOPS (randomised trial of daily oral versus pulse Cyclophosphamide as therapy for ANCA-associated Systemic Vasculitis) trial. $^{43}$ This trial was designed following a meta-analysis of three studies involving 143 patients ${ }^{44-46}$ which concluded that pulsed cyclophosphamide was more likely to achieve remission and was associated with fewer side effects than oral cyclophosphamide. ${ }^{47}$ Long-term follow-up of the CYCLOPS cohort revealed that although the proportion of participants with at least one relapse was higher in those individuals treated with pulsed cyclophosphamide, there were no differences in survival, renal function at the end of the study or adverse events between the two arms. ${ }^{48}$ However, pulsed regimens are favoured due to the reduced total dose of cyclophosphamide overall and reduced risk of bladder-related complications.

The grade of evidence for cyclophosphamide use in EGPA is lower than for GPA/MPA as no randomised controlled trials (RCTs) for the treatment of EGPA have been published. One study did compare cyclophosphamide doses: cyclophosphamide $\left(0.6 \mathrm{mg} / \mathrm{m}^{2}\right)$ was used initially every 2 weeks for a month then every 4 weeks. ${ }^{49}$ The intervention arm was given six pulses in total, while the control arm received 12 pulses. Complete remission was achieved in both groups at a similar rate $(21 / 23$ in intervention arm, 21/25 in control arm).

Antiemetic therapy should be routinely administered with intravenous cyclophosphamide. Cyclophosphamide metabolites are toxic to the urothelium and can cause haemorrhagic cystitis in the short term and malignancy in the long term. ${ }^{28} 2950$ If clinically appropriate, patients should be encouraged to drink plenty of fluids or given intravenous fluids on the day of the infusion to dilute the metabolites in the urine. Patients receiving pulse cyclophosphamide may also be given oral or intravenous 2-mercaptoethanesulfonate sodium (MESNA) which binds to acrolein, a toxic metabolite of cyclophosphamide, rendering it non-toxic. ${ }^{26}$ MESNA also retards the degradation of 4-hydroxymetabolites, further reducing the toxic 
acrolein products in the urine. MESNA may also be beneficial in patients receiving continuous oral cyclophosphamide. ${ }^{25} 2651$

Monitoring of patients receiving cyclophosphamide should follow standard protocols. ${ }^{52}$ In both modalities of administration, dose changes or discontinuation of cyclophosphamide may be necessary in the event of an acute leucopenia or a gradual fall over time. In the event of a stable leucopenia, it may be possible to maintain the immunosuppression with stringent blood monitoring. We encourage prophylaxis against infection with Pneumocystis jirovecii with trimethoprim/sulfamethoxazole (800/ $160 \mathrm{mg}$ on alternate days or $400 / 80 \mathrm{mg}$ daily) in all patients being treated with cyclophosphamide, where not contraindicated. ${ }^{53-55}$ The use of inhaled monthly pentamidine in the event of an adverse reaction or contraindication to trimethoprim/sulfamethoxazole may be useful but is not cost-effective and not routinely indicated. ${ }^{53}$ Other alternatives include dapsone and atovaquone.

Rituximab in AAV has been tested in two RCTs (RAVE (Rituximab for the Treatment of Wegener's Granulomatosis and Microscopic Polyangiitis) and RITUXVAS (an international, randomised, open label trial comparing a rituximab based regimen with a standard cyclophosphamide/azathioprine regimen in the treatment of active, 'generalised' ANCA associated vasculitis)). ${ }^{56} 57$ In both studies patients initially received high-dose glucocorticoids with subsequent dose tapering. The rituximab dose in both studies was $375 \mathrm{mg} / \mathrm{m}^{2}$ of body surface area, once a week for four infusions. In both trials, rituximab was noninferior to cyclophosphamide and appeared more effective for relapsing disease in RAVE. Details of the clinical trials in this section are available in the online supplement.

The grade of evidence for the use of rituximab in patients with EGPA is lower than for GPA/MPA. A retrospective analysis of 41 patients with EGPA who received differing regimens of rituximab found that $34 \%$ achieved complete remission at 6 months and $49 \%$ at 12 months. ${ }^{58}$

Due to high cost, rituximab use is restricted in some countries and therefore involvement of expert centres is mandated. There may be specific instances where rituximab is preferable to cyclophosphamide, for example, in patients who wish to preserve their reproductive potential. Cyclophosphamide is associated with reduced ovarian reserve, ovarian failure and male infertility. ${ }^{59-63}$ The long-term effects of rituximab on fertility have not been studied but no such concerns have been reported. In patients with severe disease, treatment should not be delayed but discussion of these issues should take place.

The task force considered appropriate a target of between 7.5 $\mathrm{mg}$ and $10 \mathrm{mg}$ of prednisolone (or equivalent) after 3 months (12 weeks) of treatment. A review of the prednisolone protocol reduction regimens published for the key trials illustrated that on average a dose of $10 \mathrm{mg}$ was achieved after 19 weeks, and a dose of $7.5 \mathrm{mg}$ after 21 weeks (figure 2). ${ }^{43} \quad 49 \begin{array}{llll}56 & 57 & 64-68\end{array}$ Therefore although a target prednisolone dose of $7.5-10 \mathrm{mg}$ is desirable by 3 months, in practice it may be 5 months before this is achieved.

The AAVs have protean manifestations and the spectrum of disease ranges from the indolent to the life-threatening. ${ }^{69-73}$ Although the evidence and thus the recommendations follow current classification systems, it is not our intention to maintain this delineation in the long term and future evidence about outcomes of phenotypes may change current labels.

\section{Statement 4}

For remission-induction of non-organ-threatening AAV we recommend treatment with a combination of glucocorticoids and either methotrexate or mycophenolate mofetil.

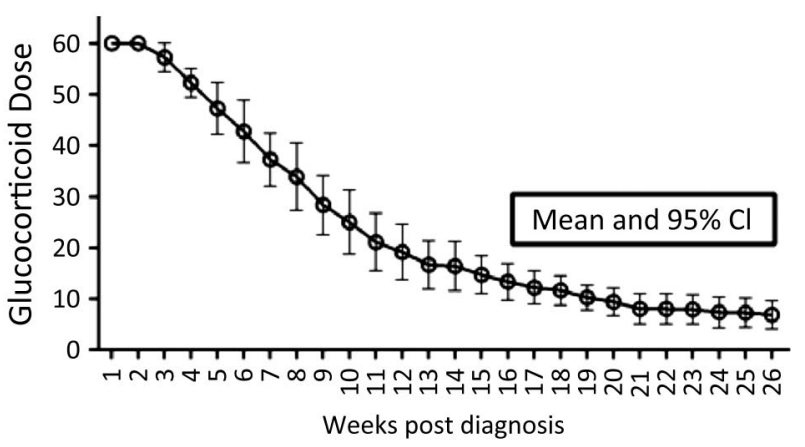

Figure 2 Protocol target prednisolone dosages in the key induction trials of antineutrophil cytoplasmic antibody associated vasculitis.

- Methotrexate

- Level of evidence 1B; grade of recommendation B; strength of vote $77 \%$.

- Mycophenolate mofetil

- Level of evidence 1B; grade of recommendation C; strength of vote $65 \%$.

The task force was keen to stress that the use of methotrexate or mycophenolate mofetil should not be used for remission induction in the following scenarios:

- Meningeal involvement

- Retro-orbital disease

- Cardiac involvement

- Mesenteric involvement

- Acute-onset mononeuritis multiplex

- Pulmonary haemorrhage of any severity

Methotrexate (20-25 mg/week, oral or parenteral) may be used as an alternative to cyclophosphamide in patients with less severe disease and in those with normal renal function. ${ }^{25} 65$ 74-81 There have been trials using either methotrexate or mycophenolate mofetil as the remission induction agent in patients with $\mathrm{AAV}^{65}$ Oral methotrexate $20-25 \mathrm{mg} /$ week was non-inferior to oral cyclophosphamide at 6 months but longterm follow-up revealed that patients treated with methotrexate had less effective disease control as compared with those treated with cyclophosphamide. ${ }^{82}$ Methotrexate should therefore be considered only for non-organ-threatening disease. Examples include the following in the absence of renal involvement

- Nasal and paranasal disease without bony involvement (erosion) or cartilage collapse or olfactory dysfunction or deafness

- Skin involvement without ulceration

- Myositis (skeletal muscle only)

- Non-cavitating pulmonary nodules/infiltrate without haemoptysis

- When cyclophosphamide or rituximab are not available or contraindicated or patient choice

The induction trials involving methotrexate are generally larger and of higher evidence grade than those using mycophenolate mofetil. To date, the two RCTs using mycophenolate mofetil have been conducted primarily in patients with MPA (of the 76 participants 75 had MPA). ${ }^{83}$ MPA often affects renal function and in such situations methotrexate would not be indicated. The trials did not include patients with lung haemorrhage or central nervous system (CNS) involvement and therefore mycophenolate mofetil should not be routinely preferred in lifethreatening situations.

Details of the clinical trials discussed in this statement are available in the online supplement. 


\section{Statement 5}

For a major relapse of organ-threatening or life-threatening disease in AAV we recommend treatment as per new disease with a combination of glucocorticoids and either cyclophosphamide OR rituximab.

- Rituximab

- level of evidence 1B for GPA and MPA; grade of recommendation A; strength of vote $94 \%$.

- level of evidence 4 for EGPA; grade of recommendation D; strength of vote $100 \%$

- Cyclophosphamide

- level of evidence 1A for GPA and MPA; grade of recommendation A; strength of vote $88 \%$.

- level of evidence 3 for EGPA; grade of recommendation C; strength of vote $88 \%$.

Most trials published on remission induction in AAV make no distinction between those participants treated for a new or relapsing presentation of their disease. It is for these reasons that the trial evidence for new or relapsing disease is often from the same studies. However, some studies have distinguished between those participants with new and relapsing disease and have stratified by this factor when randomising patients.

The largest RCT to investigate the use of rituximab for remission induction in AAV (RAVE) stratified participants by new or relapsing disease: those with relapsing disease treated with rituximab were more likely to be in disease remission at the 6-month and 12 month time points but not the 18 month follow-up visit. $^{57}$

The cumulative dose of cyclophosphamide is related to toxicity and is a particular concern with prolonged oral dosing, where cumulative doses are higher. ${ }^{85}$ For this reason the task force has favoured a greater strength of recommendation for rituximab over cyclophosphamide for relapsing disease.

The treatment of non-severe relapses in AAV with a temporary increase in the glucocorticoid dose restores disease remission in most patients but recurrent relapses within a relatively short time period remain common. ${ }^{86}$ Given these data, alternative approaches to the treatment of non-severe relapses must be considered, especially if relapses are frequent. We therefore recommend treatment with intensification or modification of the immunosuppressive remission maintenance regimen. The details of the data are available in the online supplement.

\section{Statement 6}

Plasma exchange should be considered for patients with AAV and a serum creatine level of $>500 \mu \mathrm{mol} / \mathrm{L}(5.7 \mathrm{mg} / \mathrm{dL})$ due to rapidly progressive glomerulonephritis in the setting of new or relapsing disease. Level of evidence $1 \mathrm{~B}$; grade of recommendation B; strength of vote $77 \%$.

Plasma exchange can also be considered for the treatment of severe diffuse alveolar haemorrhage. Level of evidence 3; grade of recommendation C; strength of vote $88 \%$.

PLEX use is usually reserved for patients with either severe renal impairment or those with diffuse alveolar haemorrhage. ${ }^{87-89}$ The largest trial published to date is MEPEX which recruited those individuals with either a serum creatine $>500 \mu \mathrm{mol} / \mathrm{L} \quad(5.7 \mathrm{mg} / \mathrm{dL})$ or those requiring dialysis. ${ }^{68}$ Long-term follow-up and analysis of this trial have also been published. ${ }^{90}$ PLEX appeared to be of value in preventing end-stage renal disease or death at 3 months, ${ }^{68}$ but long-term follow-up revealed no statistically significant benefit for the PLEX group. ${ }^{91}$ A prior meta-analysis had concluded that PLEX may decrease the composite end point of end stage renal disease (ESRD) or death in patients with renal vasculitis. ${ }^{92}$

However most trials of PLEX did not restrict use to individuals with a serum creatine $>500 \mu \mathrm{mol} / \mathrm{L}(5.7 \mathrm{mg} / \mathrm{dL})$. One RCT with long-term follow-up tested whether PLEX may benefit individuals with a serum creatine of $<500 \mu \mathrm{mol} / \mathrm{L}(5.7 \mathrm{mg} / \mathrm{dL}):^{93}$ after 1 month, none of the PLEX participants required haemodialysis (HD) or had worsening renal function compared with six with declining renal function and five on HD in the reference group $(p<0.05) .{ }^{93}$ Despite the improvements in renal function, there were no differences in all-cause mortality between the PLEX and reference groups after 5 years of follow-up. ${ }^{93}$ PEXIVAS (Plasma Exchange and Glucocorticoids for Treatment of Anti-Neutrophil Cytoplasm Antibody (ANCA) - Associated Vasculitis) is a global trial that is currently recruiting patients with moderate renal impairment (estimated glomerular filtration rate (eGFR) $<50 \mathrm{~mL} /$ $\mathrm{min}$ ) and aims to provide definitive answers regarding the use of PLEX in $\mathrm{AAV}^{90}$

Further details about the clinical trials and the PEXIVAS protocol are available in the online supplement.

There is also potential benefit for PLEX in patients with AAV who are also anti-glomerular basement membrane (GBM) antibody positive, particularly those in whom there is linear staining of IgG on the glomerular basement membrane, and PLEX should be performed early in such patients to improve outcome. $^{89} 94$

\section{Statement 7}

For remission maintenance of AAV we recommend treatment with a combination of low-dose glucocorticoids and either azathioprine, rituximab, methotrexate or mycophenolate mofetil.

GPA/MPA

- Azathioprine

- Level of evidence 1B for GPA and MPA; grade of recommendation A; strength of vote $94 \%$.

- Rituximab

- Level of evidence 1B for GPA and MPA; grade of recommendation A; strength of vote $59 \%$.

- Methotrexate

- Level of evidence 1B for GPA and MPA; grade of recommendation A; strength of vote $53 \%$

- Mycophenolate mofetil

- Level of evidence 1B for GPA and MPA; grade of recommendation A; strength of vote $53 \%$

EGPA

- Azathioprine

- Level of evidence 3 for EGPA; grade of recommendation C; strength of vote $77 \%$.

Long-term therapy with cyclophosphamide has been used to maintain remission in patients with $\mathrm{AAV}^{25}$ However the toxicity of long-term cyclophosphamide makes it an unattractive option. $^{28} 5029$ Azathioprine $(2 \mathrm{mg} / \mathrm{kg} /$ day $)$ is safer than oral cyclophosphamide but as effective at 18 months in preventing relapse. ${ }^{6795}$ Methotrexate (20-25 mg/kg/week) has been effectively used for maintenance therapy after induction of remission with cyclophosphamide (if the serum creatine is $<130 \mu \mathrm{mol} / \mathrm{L}$ or $1.5 \mathrm{mg} / \mathrm{dL}){ }^{96} 97$ Leflunomide $(20-30 \mathrm{mg} / \mathrm{day})$ may be more effective than methotrexate in remission maintenance but is associated with more adverse effects. ${ }^{98}$ Therefore leflunomide is considered for second line treatment in cases of intolerance to azathioprine, methotrexate, mycophenolate mofetil or 
rituximab. Early cessation of therapy is associated with an increased risk of relapse. ${ }^{65}$

The MAINRITSAN (Efficacy Study of Two Treatments in the Remission of Vasculitis) trial compared low-dose rituximab in GPA/MPA (at a fixed $500 \mathrm{mg}$ dose) to tapering dose of azathioprine for remission maintenance after induction with pulsed cyclophosphamide. ${ }^{99}$ At month 28, major relapses had occurred: 17 in the azathioprine group and 3 in the rituximab group. Renal relapses occurred in 8/17 major relapses in the azathioprine group and $0 / 3$ in the rituximab group. ${ }^{99}$

Azathioprine is preferred over mycophenolate mofetil for remission maintenance, because of the results from the IMPROVE (Mycophenolate Mofetil Versus Azathioprine for Maintenance Therapy in ANCA Associated Systemic Vasculitis) trial. ${ }^{100}$ In both groups the remission maintenance agent was reduced at two time points (after 12 months and 18 months) and withdrawn after 42 months. ${ }^{100}$ Relapses were noted in 42 participants treated with mycophenolate mofetil and in 30 participants in the azathioprine group $(\mathrm{p}<0.01)$.

The addition of trimethoprim/sulfamethoxazole $(800 / 160 \mathrm{mg}$ twice daily) to standard remission maintenance can reduce the risk of relapse in GPA. ${ }^{101}$ Although trimethoprim/sulfamethoxazole has been used as the sole remission maintenance agent in half the patients of one RCT, trimethoprim/sulfamethoxazole monotherapy may not be effective for maintenance of remission. ${ }^{101} 102$ In patients with nasal disease, treatment with topical antibiotics such as mupirocin may be considered in the presence of chronic carriage of nasal Staphylococcus aureus. ${ }^{103}$

\section{Statement 8}

We recommend that remission-maintenance therapy for AAV be continued for at least 24 months following induction of sustained remission. Level of evidence 4; grade of recommendation $\mathrm{D}$; strength of vote $75 \%$ for myeloperoxidase (MPO) persistent disease, 62\% for MPO negative disease, 100\% for PR3 persistent disease and $92 \%$ for PR3 negative disease.

No published RCTs have directly compared duration of maintenance therapy regimens. Early cessation of therapy is associated with an increased risk of relapse. ${ }^{65}{ }^{104}$ Most of the data regarding relapse risk are derived from a combination of observational cohort data and long-term follow-up from clinical trials. There are however important differences in the make-up of the participants from these sources, with many more patients with GPA likely to be present in observational cohort studies. ${ }^{105}$ In general, attempts at reduction of glucocorticoids should be made prior to tapering of the immunosuppressive agent. A meta-analysis of 13 studies (8 RCTs and 5 observational studies with 983 participants) examining the effect of duration of glucocorticoids on relapse rate concluded that continuing glucocorticoids is associated with fewer relapses. ${ }^{106}$ The pooled total estimate for the proportion of patients suffering with a relapse recruited to RCTs was $36 \%$ (95\% CI $25 \%$ to $47 \%$ ) but only $14 \%$ for those studies which continued glucocorticoids. In patients with AAV with renal involvement, worse prognosis is associated with those who have MPO-ANCA, even after adjustment for baseline factors such as age, sex and serum creatine. ${ }^{107}$ Furthermore, patients with MPO-ANCA have more severe tubulointerstitial inflammation and both $\mathrm{CD} 3(+) \mathrm{T}$ cell tubulitis and tubular atrophy are independently associated with eGFR at 12 months. ${ }^{108}$ In addition, kidney biopsies displaying sclerosis are associated with worse outcomes in AAV. ${ }^{109}$ However patients with PR3-ANCA and those with cardiovascular or lung involvement are more likely to relapse. ${ }^{72} 110$ The resultant grade for the strength of recommendation of the task force reflects the lack of data for this area. It should be noted that there was a trend to increase the duration of therapy in patients who are PR3-ANCA positive and this was reflected with median of the vote for 36 months of maintenance therapy in this particular scenario.

\section{Statement 9}

For patients with AAV refractory to remission-induction therapy we recommend switching from cyclophosphamide to rituximab or from rituximab to cyclophosphamide. These patients should be managed in close conjunction with, or referred to, an expert centre for further evaluation and potential enrolment in clinical trials. Level of evidence 3 ; grade of recommendation C; strength of vote $71 \%$.

Refractory disease is defined by EULAR as: ${ }^{111}$

- Unchanged or increased disease activity in acute AAV after 4 weeks of treatment with standard therapy in acute AAV, or

- Lack of response, defined as $<50 \%$ reduction in the disease activity score (eg, Birmingham Vasculitis Activity Score (BVAS) or BVAS/wegener's granulomatosis (WG)), after 6 weeks of treatment, or

- Chronic, persistent disease defined as presence of at least one major or three minor items on the disease activity score after $>12$ weeks of treatment.

It is important to consider why a particular patient may have refractory disease and what is driving the conclusion that they have refractory disease. Items to consider are:

- Re-evaluate the primary diagnosis; are they truly refractorydo they have AAV?

- Has the treatment regimen been optimised, that is, have target dosages for therapy been reached?

- Is this active disease or could it be damage?

- Is the present disease due to AAV or could it be due to an infection or other comorbidity or possible malignancy?

Rituximab has proven useful in patients with refractory disease, particularly those previously treated with cyclophosphamide. Patients with refractory renal disease have the greatest chance of improvement, while those with retro-orbital disease pose a particular challenge. ${ }^{58} 73112113$ Based on the results of an additional analysis of the WEGENT (Comparison of Methotrexate or Azathioprine as Maintenance Therapy for ANCA-Associated Vasculitides) trial, as a potential strategy the task force suggested a switch from pulsed to oral cyclophosphamide when rituximab is unavailable, under the guidance of an expert centre. ${ }^{114}$

In the follow-up of patients enrolled into the RAVE trial who failed to achieve the primary end point, treatment with blinded cross-over or according to best medical judgement by the trial physician lead to disease control in the majority. ${ }^{115}$ Rituximab may be better than cyclophosphamide for those participants who are PR3-ANCA positive. ${ }^{115}$

For patients who fail to achieve remission and have persistent low activity, adjunctive therapy with intravenous immunoglobulin (IVIG) may help patients achieve remission. ${ }^{116-118}$ Prior to therapy, serum immunoglobulin levels must be measured because patients with selective IgA deficiency may develop an anaphylactic reaction on receiving IVIG or a pre-existing hyperglobulinaemia may become aggravated leading to a hyperviscosity state.

Further details of the data discussed in this statement are available in the online supplement.

\section{Statement 10}

We recommend that structured clinical assessment rather than ANCA testing should inform decisions on changes in treatment 
for AAV. Level of evidence 4; grade of recommendation D; strength of vote $100 \%$.

The role of ANCA testing as a means of predicting future relapse is controversial and evolving. ${ }^{119-122}$ ANCA testing should be performed at accredited labs which take part in quality assurance testing programmes. ${ }^{123}{ }^{124}$ A negative ANCA does not rule out AAV in the appropriate clinical context of active disease. $^{125} 126$

Some studies have shown that patients in whom the ANCA titres either persist, rise fourfold or become positive have a higher incidence of relapse, while other studies did not confirm this association. ${ }^{95} 121120$ We believe that these factors should not lead to a change in therapy but more frequent clinical assessment should be considered.

Multiorgan involvement is common in AAV therefore a structured clinical assessment should be conducted in all patients. This examination may be facilitated by the use of clinical tools such as BVAS and the Vasculitis Damage Index. ${ }^{127-130}$ BVAS (V.3) was modified in 2008. ${ }^{127}$ Other validated tools include BVAS/WG, the Disease Extent Index and the Five Factor Score. ${ }^{131} 132$ These tools have a high degree of correlation and are reliable. ${ }^{133}$ Training and certification in using these tools is recommended for clinicians caring for patients with AAV.

A structured examination of the patient should be carried out at each clinic visit to detect new organ involvement, which may develop at any time in the disease course. ${ }^{134}$ Urinalysis should be performed on each patient at each visit to screen for infection, renal relapse or response, as well as bladder complications. $^{28} 5029$ During follow-up, inflammatory markers and renal function should be measured periodically (every 1-3 months) to monitor disease status. A full blood count and liver function should be performed at similar intervals to screen for drug toxicity. ${ }^{52} 67$ An acute fall in white cell count or a progressive leucopenia may require reduction or discontinuation of immunosuppressive drugs. Similarly, declining renal function may necessitate dose adjustment or alteration of immunosuppressive agents. Patients should have periodic assessment of their blood glucose while on glucocorticoid therapy. ${ }^{10}$

\section{Statement 11}

We recommend the investigation of persistent unexplained haematuria in patients with prior exposure to cyclophosphamide. Level of evidence 2B; grade of recommendation $\mathrm{C}$; strength of vote $100 \%$.

The use of cyclophosphamide is strongly associated with the risk of bladder cancer. ${ }^{28} 2950$ The use of MESNA as an uroprotective agent lowers the risk of haemorrhagic cystitis but there is no clear evidence that it protects against bladder cancer. ${ }^{85}$ Transitional cell cancer can occur within months of commencement of cyclophosphamide or many years after its discontinuation. $^{28}$ Tobacco smokers are particularly susceptible and may develop the cancer at lower doses and earlier than non-smokers. ${ }^{28}$ All patients should have periodic urinalysis for the duration of their follow-up. In the presence of haematuria confirmed on urine microscopy, an urgent urology opinion must be sought.

\section{Statement 12}

Hypoimmunoglobulinaemia has been noted after treatment with rituximab. We recommend testing of serum immunoglobulin levels prior to each course of rituximab and in patients with recurrent infection. Level of evidence 3; grade of recommendation C; strength of vote $65 \%$.

Hypoimmunoglobulinaemia is associated with repeated use of cyclophosphamide and rituximab and is dependent on the cumulative dose of the drugs used. Cyclophosphamide treatment results in a decrease in immunoglobulin (Ig) levels and subsequent rituximab treatment in patients resulted in a further decline in Ig levels. ${ }^{24}$ Surveying patients with AAV is warranted post cyclophosphamide and rituximab treatment for serum immunoglobulin concentrations and persisting hypoimmunoglobulinaemia. $^{24}$ In patients who develop this complication, involvement of a clinical immunologist is recommended. Not all patients who develop hypoimmunoglobulinaemia have infectious complications. ${ }^{135}$

Patients with AAV should be immunised against infectious disease according to local policy. It should be noted that influenza vaccination does not appear to be associated with relapse in patients with $\mathrm{AAV}^{136}{ }^{137}$ In addition patients with GPA show an adequate immune response to influenza vaccination. ${ }^{138}$ Vaccination against herpes zoster (follow local guidelines because this is a live vaccine which may be contraindicated in immunosuppressed patients), pneumococcus and influenza should be considered in patients with AAV. However one should take into account the patients' need for treatment of their AAV and of likely treatment choice for both induction and maintenance therapy. Live attenuated vaccines should be avoided whenever possible. We refer readers to the EULAR recommendation for vaccination in adult patients with autoimmune inflammatory rheumatic diseases. ${ }^{139}$

Further discussion is available in the online supplement.

\section{Statement 13}

We recommend periodic assessment of cardiovascular risk for patients with AAV. Level of evidence 2B; grade of recommendation B; strength of vote $53 \%$.

Patients with AAV are at risk of complications, both from their disease and its treatment. ${ }^{134}$ In AAV, renal, otolaryngological and treatment-related complications (cardiovascular disease, diabetes, osteoporosis and malignancy) and damage increase over time. Around a third of patients have $\geq$ five items of damage at a mean of 7 years post diagnosis. At long-term follow-up, the most commonly reported items of treatment-related complications or damage were hypertension $(41.5 \%$; $95 \%$ CI $35.6 \%$ to $47.4 \%)$, osteoporosis $(14.1 \% ; 9.9 \%$ to $18.2 \%)$, malignancy $(12.6 \% ; 8.6 \%$ to $16.6 \%)$ and diabetes $(10.4 \% ; 6.7 \%$ to $14.0 \%)$. Given that hypertension and diabetes are well known cardiovascular risk factors it is perhaps unsurprising that patients with AAV are at an increased risk for cardiovascular disease. However, the risk of cardiovascular disease appears to be greater than can be explained through traditional cardiovascular risk factors alone. A comparison of 535 participants with 5 -year follow-up from four European Vasculitis Society (EUVAS) trials revealed that within 5 years of diagnosis, 14\% of patients with GPA or MPA will have a cardiovascular event. ${ }^{140}$ This study also showed that independent determinants of cardiovascular outcome were: older age (OR 1.45, 95\% CI 1.11 to 1.90 ), diastolic hypertension (OR 1.97, 95\% CI 0.98 to 3.95) and PR3-ANCA (OR 0.39 , 95\% CI 0.20 to 0.74 ). ${ }^{140}$ Annual review of traditional Framingham risk factors is appropriate.

Patients with AAV are at risk of long-term kidney damage. Guidelines exist on the management of chronic kidney disease (CKD) such as Kidney Disease: Improving Global Outcomes (KDIGO) (http://www.kdigo.org/clinical_practice_guidelines/ pdf/CKD/KDIGO_2012_CKD_GL.pdf).

\section{Statement 14}

We recommend that patients with AAV should be given a clear verbal explanation of the nature of their disease, the treatment 
options, the side effects of treatment, and the short-term and long-term prognoses. Level of evidence 3 ; grade of recommendation C; strength of vote $88 \%$.

It is a generally accepted principle in medical practice that patients who are well informed and educated about their illness and understand it have better outcomes. An evaluation of patient education has taken place using an inpatient education programme in a tertiary referral centre, although this was not a RCT. ${ }^{141}$ Patients should be encouraged to share responsibility for dealing with their illness. ${ }^{141}$

AAV can be a bewildering and confusing illness for patients who can be very fearful when receiving a diagnosis of such an uncommon disease. Like all rare diseases there is little common experience and understanding of vasculitis so there are no readily available sources of information. Patients with rare diseases often feel isolated and alone. ${ }^{142}$

The internet can now provide access to reliable and up-to-date information and advice, and to patient support groups which provide the reassurance of peer support and the ability to share knowledge and experience. The internet can also provide incorrect, unproven and even dangerous information. It is often the least articulate and least confident who are most vulnerable and need support.

$\mathrm{AAV}$ is characteristically a relapsing disease. Each relapse may result in further morbidity so early prediction or recognition of relapse is essential. A patient who understands and is educated about the disease is frequently better able to recognise the early signs and symptoms of relapse.

\section{Statement 15}

We recommend that following the remission-induction phase of treatment, patients with AAV be assessed for the extent and ongoing impact of comorbidities associated with their diagnosis. Patients should then be advised where they might find the necessary therapies or support for these conditions. Level of evidence 4; grade of recommendation D; strength of vote $100 \%$.

$\mathrm{AAV}$ is a systemic disease with the potential to affect almost any organ. ${ }^{143}$ Patients may be left with permanent damage to kidneys, lungs and respiratory tract, heart, peripheral and central nervous system, total or partial loss of sight or hearing. ${ }^{144} 145$ Patients may lose digits or limbs or be left with facial disfigurement (like a saddle-nose) or severe skin scarring. ${ }^{27}$ Severe fatigue, muscle weakness and chronic pain are frequent direct consequences of AAV. ${ }^{146}{ }^{147}$ Side effects of treatment can be serious, even life-threatening. ${ }^{148}$

The consequences of AAV may have a serious impact on education, employment prospects and job retention. ${ }^{149}$ Personal and social relationships may be seriously disrupted, sometimes resulting in the total breakdown of family bonds. These factors may contribute to depression as a secondary consequence of $\mathrm{AAV}^{150} 151$

AAV is a controllable but currently incurable lifelong illness. Treating clinicians need to be aware that AAV often has longterm lifestyle consequences. A 'holistic' approach to treatment and ongoing care should be adopted.

\section{DISCUSSION}

\section{Implementation of these recommendations}

The recommendations have been based on an extensive literature search. In the absence of evidence, statements have been based on the opinion and practice of experts from 12 countries (Czech Republic, France, Germany, Ireland, Italy, Netherlands, Spain, Sweden, Switzerland, Turkey, UK and USA). The application of internationally accepted grading criteria prevents us

\section{Box 1 Research agenda}

- Diagnostic and classification criteria for ANCA-associated vasculitis (AAV).

- Identification of biomarkers for AAV.

- Adjunctive plasma exchange-indications for use including serum creatinine cut-off.

- Adequately powered clinical trials of novel biological agents for the treatment of refractory AAV.

- Adequately powered randomised controlled trials in eosinophilic granulomatosis with polyangiitis.

- Long-term outcome studies in AAV.

from supporting some of the statements with stronger grades. The project has also led the committee to propose a research agenda for AAV (see box 1). These recommendations have been multidisciplinary with inputs from rheumatologists, internists, renal physicians and also from a clinical immunologist, an otorhinolaryngologist, a chest physician, an ophthalmologist, a vasculitis nurse and a patient with vasculitis. In addition to these recommendations, we have also produced advice on AAV involving the eye and the nose (online supplement) and a lay summary for patients and relatives (online supplement).

The previous recommendations were published in 2009 and importantly had a wider remit, covering small and medium vessel vasculitis and not just $\mathrm{AAV}^{10}$ Readers are encouraged to refer to them for treatment decisions on: mixed essential cryoglobulinaemic vasculitis (non-viral), the use of antiviral therapy for the treatment of hepatitis $\mathrm{C}$ associated cryoglobulinaemic vasculitis and antiviral therapy, PLEX and glucocorticoids for hepatitis B-associated polyarteritis nodosa (PAN). Ultimately the treatment aim of viral-associated cryoglobulinaemic vasculitis should be to treat the underlying viral disease according to current best management strategies.

The current recommendations provide a framework of practice which have updated the previous recommendations and should apply to the majority of patients with AAV. Although once again 15 statements have been formulated; some have been changed and some have been combined: for example there is no longer a separation of glucocorticoids as they are used in conjunction with other immunosuppressive agents. For remission maintenance the voting of the task force reveals that azathioprine is the preferred option over other immunosuppressive agents. In specific situations, where a less aggressive induction regimen has to be preferred-methotrexate or mycophenolate mofetil may be recommended. The task force appreciates that the induction trials involving methotrexate are larger and of higher evidence grade than those using mycophenolate mofetil. We have been explicit in the limited scenarios where methotrexate or mycophenolate mofetil may be justified.

Each statement should be an opportunity for auditing clinical practice (an audit tool has been produced-see online supplement). In addition these current recommendations have produced algorithms which provide clear and concise information for the management of AAV (see figure 1).

These recommendations have also been voted on by the EUVAS membership the results of which are available as a supplementary online file (see online supplement). The results of the EUVAS vote are largely in agreement with the strength of recommendation vote by the task force. There are differences particularly when there are a number of options available and 
the resultant vote may represent the diversity of the EUVAS membership. Importantly task force members who are also members of EUVAS did not vote in the EUVAS survey. Recommendations for clinical management need periodic updating and because of the many advances and ongoing research in this field, this group recommends an update of these recommendations should be conducted in 3 years.

\section{Author affiliations}

${ }^{1}$ Department of Rheumatology, Norfolk and Norwich University Hospital, Norwich, UK

${ }^{2}$ Norwich Medical School, University of East Anglia, Norwich, UK

${ }^{3}$ Department of Rheumatology, Ipswich Hospital NHS Trust, Ipswich, Suffolk, UK

${ }^{4}$ Department of Pathology, Leiden University Medical Center, Leiden, The Netherlands

${ }^{5}$ Vasculitis Research Unit, Department of Autoimmune Diseases, Hospital Clínic, University of Barcelona, Institut d'Investigacions Biomèdiques August Pi i Sunyer (IDIBAPS), Barcelona, Spain

${ }^{6}$ Assistance Publique-Hôpitaux de Paris, Department of Pulmonology, Bichat-Claude Bernard University Hospital, Paris, France

${ }^{7}$ Immunologie-Zentrum Zürich, Zürich, Switzerland

${ }^{8}$ Vaskulits-Zentrum Süd, Klinik für Innere Medizin, Rheumatologie und Immunologie, Kreiskliniken Esslingen, Kirchheim-Teck, Germany

${ }^{9}$ Rheumazentrum Schleswig-Holstein Mitte, Neumünster, Germany

${ }^{10}$ Department of Otorhinolaryngology, Head and Neck Surgery, University of Kiel, Kiel, Germany

${ }^{11}$ Trinity Health Kidney Centre, Tallaght Hospital, Dublin, Ireland

${ }^{12}$ Nuffield Department of Orthopaedics Rheumatology and Musculoskeletal Sciences, Botnar Research Centre, University of Oxford, Oxford, United Kingdom

${ }^{13}$ Department of Internal Medicine, Hôpital Saint-Louis, Université Paris 7 René Diderot, Paris, France

${ }^{14}$ Division of Rheumatology and the Department of Biostatistics and Epidemiology, University of Pennsylvania, Philadelphia, Pennsylvania, USA

${ }^{15}$ Vasculitis UK, West Bank House, Winster, Matlock, UK

${ }^{16}$ Department of Medical and Health Sciences, Linköping University, Linköping, Sweden

${ }^{17}$ Department of Nephrology, Linköping University, Linköping, Sweden

${ }^{18}$ Department of Nephrology, 1st School of Medicine, Charles University, Prague,

Czech Republic

${ }^{19}$ Department of Nephrology, Lund University, Skåne University Hospital, Lund and Malmö, Sweden

${ }^{20}$ Nephrology Unit, University Hospital of Parma, Parma, Italy

${ }^{21}$ Department of Ophthalmology, School of Medicine, Ankara University, Ankara, Turkey

${ }^{22}$ Lupus and Vasculitis Unit, Addenbrooke's Hospital, Cambridge, UK

Acknowledgements The authors thank Ms Karly Graham for proof reading.

Contributors All authors formed part of the task force that appraised the evidence and made recommendations for the management of ANCA-associated vasculitis. All authors have contributed to editing of drafts of the main document and associated supplementary materials.

Funding This work was supported by and commissioned by the European League Against Rheumatism (EULAR) and the European Renal Association-European Dialysis and Transplant Association (ERA-EDTA).

Competing interests DRJ has received research grants and consulting fees from Roche/Genentech.

Provenance and peer review Not commissioned; externally peer reviewed.

\section{REFERENCES}

1 Jennette JC, Falk RJ, Bacon PA, et al. 2012 revised International Chapel Hill Consensus Conference Nomenclature of Vasculitides. Arthritis Rheum 2013:65:1-11.

2 Watts RA, Al-Taiar A, Scott DG, et al. Prevalence and incidence of Wegener's granulomatosis in the UK general practice research database. Arthritis Rheum 2009;61:1412-16

3 Mohammad AJ, Jacobsson LT, Westman KW, et al. Incidence and survival rates in Wegener's granulomatosis, microscopic polyangiitis, Churg-Strauss syndrome and polyarteritis nodosa. Rheumatology (Oxford) 2009;48:1560-5.

4 Takala JH, Kautiainen H, Malmberg H, et al. Incidence of Wegener's granulomatosis in Finland 1981-2000. Clin Exp Rheumatol 2008;26(3 Suppl 49): S81-85.

5 Reinhold-Keller E, Herlyn $\mathrm{K}$, Wagner-Bastmeyer R, et al. Stable incidence of primary systemic vasculitides over five years: results from the German vasculitis register. Arthritis Rheum 2005;53:93-9.
6 Gibelin A, Maldini C, Mahr A. Epidemiology and etiology of wegener granulomatosis, microscopic polyangiitis, churg-strauss syndrome and goodpasture syndrome: vasculitides with frequent lung involvement. Semin Respir Crit Care Med 2011;32:264-73.

7 Mahr A, Guillevin L, Poissonnet M, et al. Prevalences of polyarteritis nodosa, microscopic polyangiitis, Wegener's granulomatosis, and Churg-Strauss syndrome in a French urban multiethnic population in 2000: a capture-recapture estimate. Arthritis Rheum 2004:51:92-9.

8 Ormerod AS, Cook MC. Epidemiology of primary systemic vasculitis in The Australian Capital Territory and south-eastern New South Wales. Intern Med J 2008:38:816-23.

9 Mukhtyar C, Flossmann O, Hellmich B, et al. Outcomes from studies of antineutrophil cytoplasm antibody associated vasculitis: a systematic review by the European League Against Rheumatism systemic vasculitis task force. Ann Rheum Dis 2008:67:1004-10.

10 Mukhtyar C, Guillevin L, Cid MC, et al. EULAR recommendations for the management of primary small and medium vessel vasculitis. Ann Rheum Dis 2009;68:310-7.

11 Dougados M, Betteridge N, Burmester GR, et al. EULAR standardised operating procedures for the elaboration, evaluation, dissemination, and implementation of recommendations endorsed by the EULAR standing committees. Ann Rheum Dis 2004:63:1172-6.

12 Boomsma MM, Bijl M, Stegeman CA, et al. Patients' perceptions of the effects of systemic lupus erythematosus on health, function, income, and interpersonal relationships: a comparison with Wegener's granulomatosis. Arthritis Rheum 2002;47:196-201.

13 Koutantji M, Harrold E, Lane SE, et al. Investigation of quality of life, mood, pain, disability, and disease status in primary systemic vasculitis. Arthritis Rheum 2003:49:826-37.

14 Anderson K, Klassen J, Stewart SA, et al. Does geographic location affect incidence of ANCA-associated renal vasculitis in northern Saskatchewan, Canada? Rheumatology 2013;52:1840-4.

15 Guillevin L, Durand-Gasselin B, Cevallos R, et al. Microscopic polyangiitis: Clinical and laboratory findings in eighty-five patients. Arthritis Rheum 1999:42:421-30.

16 Carruthers DM, Watts RA, Symmons DPM, et al. Wegener's granulomatosisincreased incidence or increased recognition? Br J Rheumatol 1996;35:142-5.

17 Hoffman GS, Thomas-Golbanov CK, Chan J, et al. Treatment of subglottic stenosis, due to Wegener's granulomatosis, with intralesional corticosteroids and dilation. I Rheumatol 2003;30:1017-21.

18 Langford CA, Sneller MC, Hallahan CW, et al. Clinical features and therapeutic management of subglottic stenosis in patients with Wegener's granulomatosis. Arthritis Rheum 1996;39:1754-60.

19 Elmedhem A, Adu D, Savage COS. Relapse rate and outcome of ANCA-associated small vessel vasculitis after transplantation. Nephrol Dial Transplant 2003;18:1001-4.

20 Rahmattulla C, de Lind van Wijngaarden RA, Berden AE, et al. Renal function and ear, nose, throat involvement in anti-neutrophil cytoplasmic antibody-associated vasculitis: prospective data from the European Vasculitis Society clinical trials. Rheumatology (Oxford) 2015;54:899-907.

21 Homma S, Suzuki A, Sato K. Pulmonary involvement in ANCA-associated vasculitis from the view of the pulmonologist. Clin Exp Nephrol 2013;17: $667-71$.

22 Le Berre L, Dufay A, Cantarovich D, et al. Early and irreversible recurrence MPO-ANCA-positive glomerulonephritis after renal transplantation. Clin Nephrol 2015:83:357-62.

23 Schokkenbroek AA, Franssen CF, Dikkers FG. Dilatation tracheoscopy for laryngeal and tracheal stenosis in patients with Wegener's granulomatosis. Eur Arch Otorhinolaryngol 2008;265:549-55.

24 Venhoff N, Effelsberg NM, Salzer U, et al. Impact of rituximab on immunoglobulin concentrations and $B$ cell numbers after cyclophosphamide treatment in patients with ANCA-associated vasculitides. PLOS ONE 2012;7:e37626.

25 Hoffman GS, Kerr GS, Leavitt RY, et al. Wegener granulomatosis: an analysis of 158 patients. Ann Intern Med 1992;116:488-98.

26 Reinhold-Keller E, Beuge N, Latza U, et al. An interdisciplinary approach to the care of patients with Wegener's granulomatosis: long-term outcome in 155 patients. Arthritis Rheum 2000;43:1021-32.

27 Flossmann O, Berden A, de Groot K, et al. Long-term patient survival in ANCA-associated vasculitis. Ann Rheum Dis 2011;70:488-94.

28 Talar-Williams C, Hijazi YM, Walther MM, et al. Cyclophosphamide-induced cystitis and bladder cancer in patients with Wegener granulomatosis. Ann Intern Med 1996;124:477-84.

29 Knight A, Askling J, Granath F, et al. Urinary bladder cancer in Wegener's granulomatosis: risks and relation to cyclophosphamide. Ann Rheum Dis 2004:63:1307-11.

30 Moosig F, Bremer JP, Hellmich B, et al. A vasculitis centre based management strategy leads to improved outcome in eosinophilic granulomatosis and polyangiitis (Churg-Strauss, EGPA): monocentric experiences in 150 patients. Ann Rheum Dis 2013;72:1011-17 
31 Aasarød K, Bostad L, Hammerstrøm J, et al. Renal histopathology and clinical course in 94 patients with Wegener's granulomatosis. Nephrol Dial Transplant 2001;16:953-60.

32 Schnabel A, Holl-Ulrich K, Dalhoff K, et al. Efficacy of transbronchial biopsy in pulmonary vaculitides. Eur Respir J 1997;10:2738-43.

33 Jennings $C R$, Jones NS, Dugar J, et al. Wegener's granulomatosis-a review of diagnosis and treatment in 53 subjects. Rhinology 1998;36:188-91.

34 Devaney KO, Travis WD, Hoffman G, et al. Interpretation of head and neck biopsies in Wegener's granulomatosis. A pathologic study of 126 biopsies in 70 patients. Am J Surg Pathol 1990;14:555-64.

35 Travis WD, Hoffman GS, Leavitt RY, et al. Surgical pathology of the lung in Wegener's granulomatosis. Review of 87 open lung biopsies from 67 patients. Am J Surg Pathol 1991;15:315-33.

36 Prasad N, Kumar S, Manjunath R, et al. Real-time ultrasound-guided percutaneous renal biopsy with needle guide by nephrologists decreases post-biopsy complications. Clin Kidney J 2015;8:151-6.

37 Basic-Jukic N, Kes P, Glavas-Boras S, et al. Complications of therapeutic plasma exchange: experience with 4857 treatments. Ther Apher Dial 2005;9:391-5.

38 Corapi KM, Chen JL, Balk EM, et al. Bleeding complications of native kidney biopsy: a systematic review and meta-analysis. Am J Kidney Dis 2012:60:62-73.

39 Berden AE, Ferrario F, Hagen EC, et al. Histopathologic classification of ANCA-associated glomerulonephritis. J Am Soc Nephrol 2010;21:1628-36.

40 Chang DY, Wu LH, Liu G, et al. Re-evaluation of the histopathologic classification of ANCA-associated glomerulonephritis: a study of 121 patients in a single center Nephrol Dial Transplant 2012;27:2343-9.

41 Noone DG, Twilt M, Hayes WN, et al. The new histopathologic classification of ANCA-associated GN and its association with renal outcomes in childhood. Clin J Am Soc Nephrol 2014;9:1684-91.

42 Novack SN, Pearson CM. Cyclophosphamide therapy in Wegener's Granulomatosis. N Engl J Med 1971;284:938-42.

43 de Groot K, Harper L, Jayne DR, et al. Pulse versus daily oral cyclophosphamide for induction of remission in antineutrophil cytoplasmic antibody-associated vasculitis: a randomized trial. Ann Intern Med 2009;150:670-80.

44 Adu D, Pall A, Luqmani RA, et al. Controlled trial of pulse versus continuous prednisolone and cyclophosphamide in the treatment of systemic vasculitis. QJM 1997:90:401-9.

45 Haubitz M, Frei U, Rother U, et al. Cyclophosphamide pulse therapy in Wegener's granulomatosis. Nephrol Dial Transplant 1991;6:531-4.

46 Guillevin L, Cordier JF, Lhote F, et al. A prospective, multicenter, randomized trial comparing steroids and pulse cyclophosphamide versus steroids and oral cyclophosphamide in the treatment of generalized Wegener's granulomatosis. Arthritis Rheum 1997;40:2187-98. doi:10.1002/1529-0131(199712) 40:12<2187::AID-ART12>3.0.CO;2-H

47 De Groot K, Adu D, Savage COS. The value of pulse cyclophosphamide in ANCA-associated vasculitis: Meta-analysis and critical review. Nephrol Dial Transplant 2001;16:2018-27.

48 Harper L, Morgan MD, Walsh M, et al. Pulse versus daily oral cyclophosphamide for induction of remission in ANCA-associated vasculitis: long-term follow-up. Ann Rheum Dis 2012;71:955-60.

49 Cohen P, Pagnoux C, Mahr A, et al. Churg-Strauss syndrome with poor-prognosis factors: a prospective multicenter trial comparing glucocorticoids and six or twelve cyclophosphamide pulses in forty-eight patients. Arthritis Rheum 2007;57:686-93.

50 Stillwell TJ, Benson RC Jr, DeRemee RA, et al. Cyclophosphamide-induced bladder toxicity in Wegener's granulomatosis. Arthritis Rheum 1988;31:465-70.

51 Hellmich B, Kausch I, Doehn C, et al. Urinary bladder cancer in Wegener's granulomatosis: is it more than cyclophosphamide?. Ann Rheum Dis 2004;63:1183-5.

52 Chakravarty K, McDonald $H$, Pullar T, et al. BSR/BHPR guideline for disease-modifying anti-rheumatic drug (DMARD) therapy in consultation with the British Association of Dermatologists. Rheumatology (Oxford) 2008;47:924-5.

53 Chung JB, Armstrong K, Schwartz JS, et al. Cost-effectiveness of prophylaxis against pneumocystis carinii pneumonia in patients with Wegener's granulomatosis undergoing immunosuppressive therapy. Arthritis Rheum 2000;43:1841-8.

54 Ognibene FP, Shelhamer JH, Hoffman GS, et al. Pneumocystis carinii pneumonia: A major complication of immunosuppressive therapy in patients with Wegener's granulomatosis. Am J Respir Crit Care Med 1995;151(3 Pt I):795-9.

55 Jarrousse B, Guillevin L, Bindi $P$, et al. Increased risk of Pneumocystis carinii pneumonia in patients with Wegener's granulomatosis. Clin Exp Rheumatol 1993;11:615-21.

56 Jones RB, Tervaert JW, Hauser T, et al. Rituximab versus cyclophosphamide in ANCA-associated renal vasculitis. N Engl J Med 2010;363:211-20.

57 Stone JH, Merkel PA, Spiera R, et al. Rituximab versus cyclophosphamide for ANCA-associated vasculitis. N Engl J Med 2010;363:221-32.

58 Mohammad AJ, Hot A, Arndt $F$, et al. Rituximab for the treatment of eosinophilic granulomatosis with polyangiitis (Churg-Strauss). Ann Rheum Dis 2016;75:396-401.
59 Koyama H, Wada T, Nishizawa Y, et al. Cyclophosphamide-induced ovarian failure and its therapeutic significance in patients with breast cancer. Cancer 1977;39:1403-9.

60 Mersereau J, Dooley MA. Gonadal failure with cyclophosphamide therapy for lupus nephritis: advances in fertility preservation. Rheum Dis Clin North Am 2010;36:99-108, viii.

61 Silva CA, Hallak J, Pasqualotto FF, et al. Gonadal function in Male adolescents and young males with juvenile onset systemic lupus erythematosus. I Rheumatol 2002;29:2000-5.

62 Schrader M, Heicappell R, Müller M, et al. Impact of chemotherapy on Male fertility. Onkologie 2001;24:326-30.

63 Clowse ME, Copland SC, Hsieh TC, et al. Ovarian reserve diminished by oral cyclophosphamide therapy for granulomatosis with polyangiitis (Wegener's). Arthritis Care Res (Hoboken) 2011;63:1777-81.

64 Stassen PM, Tervaert JWC, Stegeman CA. Induction of remission in active anti-neutrophil cytoplasmic antibody-associated vasculitis with mycophenolate mofetil in patients who cannot be treated with cyclophosphamide. Ann Rheum Dis 2007;66:798-802.

65 De Groot K, Rasmussen N, Bacon PA, et al. Randomized trial of cyclophosphamide versus methotrexate for induction of remission in early systemic antineutrophil cytoplasmic antibody-associated vasculitis. Arthritis Rheum 2005;52:2461-9.

66 Mansfield N, Hamour S, Habib AM, et al. Prolonged disease-free remission following rituximab and low-dose cyclophosphamide therapy for renal ANCA-associated vasculitis. Nephrol Dial Transplant 2011;26:3280-6.

67 Jayne $D$, Rasmussen N, Andrassy K, et al. A randomized trial of maintenance therapy for vasculitis associated with antineutrophil cytoplasmic autoantibodies. N Engl J Med 2003;349:36-44.

68 Jayne DRW, Gaskin G, Rasmussen N, et al. Randomized trial of plasma exchange or high-dosage methylprednisolone as adjunctive therapy for severe renal vasculitis. J Am Soc Nephrol 2007;18:2180-8.

69 Sokołowska B, Szczeklik W, Mastalerz L, et al. Effect of delayed diagnosis on disease course and management of Churg-Strauss syndrome: a retrospective study. Clin Rheumatol 2013;32:349-54.

70 Takala JH, Kautiainen $\mathrm{H}$, Malmberg $\mathrm{H}$, et al. Wegener's granulomatosis in Finland in 1981-2000: clinical presentation and diagnostic delay. Scand J Rheumatol 2008;37:435-8.

71 Howse M, Main J. Simple urine testing could avoid delay in the diagnosis of rapidly progressive glomerulonephritis. Postgrad Med J 1997;73:808-9.

72 Walsh M, Flossmann 0, Berden A, et al. Risk factors for relapse of antineutrophil cytoplasmic antibody-associated vasculitis. Arthritis Rheum 2012;64:542-8.

73 Holle JU, Voigt C, Both M, et al. Orbital masses in granulomatosis with polyangiitis are associated with a refractory course and a high burden of local damage. Rheumatology (Oxford) 2013;52:875-82.

74 Hoffman GS, Leavitt RY, Kerr GS, et al. The treatment of Wegener's granulomatosis with glucocorticoids and methotrexate. Arthritis Rheum 1992:35:1322-9.

75 Sneller MC, Hoffman GS, Talar-Williams C, et al. An analysis of forty-two Wegener's granulomatosis patients treated with methotrexate and prednisone. Arthritis Rheum 1995;38:608-13.

76 Stone JH, Tun W, Hellman DB. Treatment of non-life threatening Wegener's granulomatosis with methotrexate and daily prednisone as the initial therapy of choice. J Rheumatol 1999:26:1134-9.

77 Langford CA, Talar-Williams C, Sneller MC. Use of methotrexate and glucocorticoids in the treatment of Wegener's granulomatosis. Long-term renal outcome in patients with glomerulonephritis. Arthritis Rheum 2000;43:1836-40.

78 Stone JH. Etanercept plus standard therapy for Wegener's granulomatosis. N Engl J Med 2005:352:351-61.

79 De Groot K, Mühler M, Reinhold-Keller $E$, et al. Induction of remission in Wegener's granulomatosis with low dose methotrexate. J Rheumatol 1998;25:492-5.

80 Metzler C, Hellmich B, Gause A, et al. Churg Strauss syndrome-successful induction of remission with methotrexate and unexpected high cardiac and pulmonary relapse ratio during maintenance treatment. Clin Exp Rheumatol 2004;22(6 SUPPL.):S-52-61.

81 Metzler C, Hellmich B, Gause A, et al. Churg Strauss syndrome--successful induction of remission with methotrexate and unexpected high cardiac and pulmonary relapse ratio during maintenance treatment. Clin Exp Rheumatol 2004;22(Suppl 36):S52-61.

82 Faurschou M, Westman K, Rasmussen N, et al. Brief Report: long-term outcome of a randomized clinical trial comparing methotrexate to cyclophosphamide for remission induction in early systemic antineutrophil cytoplasmic antibodyassociated vasculitis. Arthritis Rheum 2012;64:3472-7.

83 Hu W, Liu C, Xie H, et al. Mycophenolate mofetil versus cyclophosphamide for inducing remission of ANCA vasculitis with moderate renal involvement. Nephrol Dial Transplant 2008;23:1307-12.

84 Han F, Liu G, Zhang X, et al. Effects of mycophenolate mofetil combined with corticosteroids for induction therapy of microscopic polyangiitis. Am J Nephrol 2011;33:185-92. 
85 Monach PA, Arnold LM, Merkel PA. Incidence and prevention of bladder toxicity from cyclophosphamide in the treatment of rheumatic diseases: A data-driven review. Arthritis Rheum 2010;62:9-21.

86 Miloslavsky EM, Specks U, Merkel PA, et al. Outcomes of nonsevere relapses in antineutrophil cytoplasmic antibody-associated vasculitis treated with glucocorticoids. Arthritis Rheumatol 2015;67:1629-36.

87 Klemmer PJ, Chalermskulrat W, Reif MS, et al. Plasmapheresis Therapy for Diffuse Alveolar Hemorrhage in Patients with Small-Vessel Vasculitis. Am J Kidney Dis 2003;42:1149-53

88 Levy JB, Turner AN, Rees AJ, et al. Long-term outcome of anti-glomerular basement membrane antibody disease treated with plasma exchange and immunosuppression. Ann Intern Med 2001;134:1033-42.

89 Levy JB, Hammad T, Coulthart A, et al. Clinical features and outcome of patients with both ANCA and anti-GBM antibodies. Kidney Int 2004;66:1535-40.

90 Walsh M, Merkel PA, Peh CA, et al. Plasma exchange and glucocorticoid dosing in the treatment of anti-neutrophil cytoplasm antibody associated vasculitis (PEXIVAS): protocol for a randomized controlled trial. Trials 2013:14:73.

91 Walsh M, Casian A, Flossmann 0, et al. Long-term follow-up of patients with severe ANCA-associated vasculitis comparing plasma exchange to intravenous methylprednisolone treatment is unclear. Kidney Int 2013;84:397-402.

92 Walsh M, Catapano F, Szpirt W, et al. Plasma exchange for renal vasculitis and idiopathic rapidly progressive glomerulonephritis: a meta-analysis. Am J Kidney Dis 2011;57:566-74

93 Szpirt WM, Heaf JG, Petersen J. Plasma exchange for induction and cyclosporine A for maintenance of remission in Wegener's granulomatosis - a clinical randomized controlled trial. Nephrol Dial Transplant 2011;26:206-13.

94 Peters DK, Rees AJ, Lockwood CM, et al. Treatment and prognosis in antibasement membrane antibody-mediated nephritis. Transplant Proc 1982;14:513-21.

95 Slot MC, Tervaert JWC, Boomsma MM, et al. Positive Classic Antineutrophil Cytoplasmic Antibody (C-ANCA) Titer at Switch to Azathioprine Therapy Associated with Relapse in Proteinase 3-Related Vasculitis. Arthritis Rheum 2004:51:269-73.

96 Langford CA, Talar-Williams C, Barron KS, et al. Use of a cyclophosphamide-induction methotrexate-maintenance regimen for the treatment of Wegener's granulomatosis: extended follow-up and rate of relapse. Am J Med 2003;114:463-9.

97 Reinhold-Keller E, Fink COE, Herlyn K, et al. High rate of renal relapse in 71 patients with Wegener's granulomatosis under maintenance of remission with low-dose methotrexate. Arthritis Rheum 2002;47:326-32.

98 Metzler C, Miehle N, Manger K, et al. Elevated relapse rate under oral methotrexate versus leflunomide for maintenance of remission in Wegener's granulomatosis. Rheumatology (Oxford) 2007;46:1087-91.

99 Guillevin L, Pagnoux C, Karras A, et al. Rituximab versus azathioprine for maintenance in ANCA-associated vasculitis. N Engl J Med 2014:371:1771-80.

100 Hiemstra TF, Walsh M, Mahr A, et al. Mycophenolate mofetil vs azathioprine for remission maintenance in antineutrophil cytoplasmic antibody-associated vasculitis: a randomized controlled trial. JAMA 2010;304:2381-8.

101 Stegeman CA, Tervaert JWC, De Jong PE, et al. Trimethoprim-sulfamethoxazole (Co-trimoxazole) for the prevention of relapses of Wegener's granulomatosis. $N$ Engl J Med 1996;335:16-20.

102 Reinhold-Keller E, De Groot K, Rudert H, et al. Response to trimethoprim/ sulfamethoxazole in Wegener's granulomatosis depends on the phase of disease. QJM 1996;89:15-23.

103 Stegeman CA, Cohen Tervaert JW, Sluiter WJ, et al. Association of chronic nasal carriage of Staphylococcus aureus and higher relapse rates in Wegener granulomatosis. Ann Intern Med 1994;120:12-17.

104 Springer J, Nutter B, Langford CA, et al. Granulomatosis with polyangiitis (Wegener's): impact of maintenance therapy duration. Medicine (Baltimore) 2014:93:82-90.

105 Pagnoux C, Carette S, Khalidi NA, et al. Comparability of patients with ANCA-associated vasculitis enrolled in clinical trials or in observational cohorts. Clin Exp Rheumatol 2015:33(2 Suppl 89):77-83.

106 Walsh M, Merkel PA, Mahr A, et al. Effects of duration of glucocorticoid therapy on relapse rate in antineutrophil cytoplasmic antibody-associated vasculitis: a meta-analysis. Arthritis Care Res (Hoboken) 2010;62:1166-73.

107 Mohammad AJ, Segelmark M. A population-based study showing better renal prognosis for proteinase 3 antineutrophil cytoplasmic antibody (ANCA)-associated nephritis versus myeloperoxidase ANCA-associated nephritis. J Rheumatol 2014;41:1366-73

108 Berden $A E$, Jones RB, Erasmus DD, et al. Tubular lesions predict renal outcome in antineutrophil cytoplasmic antibody-associated glomerulonephritis after rituximab therapy. J Am Soc Nephrol 2012;23:313-21.

109 Ford SL, Polkinghorne KR, Longano A, et al. Histopathologic and clinical predictors of kidney outcomes in ANCA-associated vasculitis. Am J Kidney Dis 2014:63:227-35.
110 Pagnoux C, Hogan SL, Chin H, et al. Predictors of treatment resistance and relapse in antineutrophil cytoplasmic antibody-associated small-vessel vasculitis: comparison of two independent cohorts. Arthritis Rheum 2008;58:2908-18.

111 Hellmich B, Flossmann 0, Gross WL, et al. EULAR recommendations for conducting clinical studies and/or clinical trials in systemic vasculitis: focus on anti-neutrophil cytoplasm antibody-associated vasculitis. Ann Rheum Dis 2007;66:605-17.

112 Pullerits R, Ljevak M, Vikgren J, et al. Off-trial evaluation of the B cell-targeting treatment in the refractory cases of antineutrophil cytoplasmic antibodies (ANCA)-associated vasculitis: long-term follow-up from a single centre. Scand J Immunol 2012:76:411-20.

113 Cartin-Ceba R, Keogh KA, Specks U, et al. Rituximab for the treatment of Churg-Strauss syndrome with renal involvement. Nephrol Dial Transplant 2011;26:2865-71.

114 Seror R, Pagnoux C, Ruivard M, et al. Treatment strategies and outcome of induction-refractory Wegener's granulomatosis or microscopic polyangiitis: analysis of 32 patients with first-line induction-refractory disease in the WEGENT trial. Ann Rheum Dis 2010;69:2125-30.

115 Miloslavsky EM, Specks U, Merkel PA, et al. Clinical outcomes of remission induction therapy for severe antineutrophil cytoplasmic antibody-associated vasculitis. Arthritis Rheum 2013:65:2441-9.

116 Muso E, Ito-Ihara T, Ono T, et al. Intravenous immunoglobulin (IVIg) therapy in MPO-ANCA related polyangiitis with rapidly progressive glomerulonephritis in Japan. Jpn J Infect Dis 2004;57:S17-18.

117 Jayne DRW, Chapel $H$, Adu D, et al. Intravenous immunoglobulin for ANCA-associated systemic vasculitis with persistent disease activity. QJM 2000;93:433-9.

118 Fortin PM, Tejani AM, Bassett K, et al. Intravenous immunoglobulin as adjuvant therapy for Wegener's granulomatosis. Cochrane Database Syst Rev 2013;1:CD007057.

119 Tomasson G, Grayson PC, Mahr AD, et al. Value of ANCA measurements during remission to predict a relapse of ANCA-associated vasculitis-a meta-analysis. Rheumatology (Oxford) 2012:51:100-9.

120 Finkielman JD, Merkel PA, Schroeder D, et al. Antiproteinase 3 antineutrophil cytoplasmic antibodies and disease activity in Wegener granulomatosis. Ann Intern Med 2007;147:611-19.

121 Boomsma MM, Stegeman CA, van der Leij MJ, et al. Prediction of relapses in Wegener's granulomatosis by measurement of antineutrophil cytoplasmic antibody levels: a prospective study. Arthritis Rheum 2000;43:2025-33.

122 Birck R, Schmitt WH, Kaelsch IA, et al. Serial ANCA determinations for monitoring disease activity in patients with ANCA-associated vasculitis: Systematic review. Am J Kidney Dis 2006:47:15-23.

123 Savige J, Gillis D, Benson E, et al. International consensus statement on testing and reporting of antineutrophil cytoplasmic antibodies (ANCA). Am J Clin Pathol 1999;111:507-13.

124 Savige J, Dimech W, Fritzler $\mathrm{M}$, et al. Addendum to the International Consensus Statement on testing and reporting of Antineutrophil Cytoplasmic antibodies. Quality control guidelines, comments, and recommendations for testing in other autoimmune diseases. Am J Clin Pathol 2003;120:312-18.

125 Finkielman JD, Lee AS, Hummel AM, et al. ANCA are detectable in nearly all patients with active severe Wegener's granulomatosis. Am J Med 2007;120:643.e9-14.

126 Stone JH. Limited versus severe Wegener's granulomatosis: baseline data on patients in the Wegener's granulomatosis etanercept trial. Arthritis Rheum 2003:48:2299-309.

127 Mukhtyar C, Lee R, Brown D, et al. Modification and validation of the Birmingham Vasculitis Activity Score (version 3). Ann Rheum Dis 2009;68:1827-32.

128 Suppiah R, Mukhtyar C, Flossmann 0, et al. A cross-sectional study of the Birmingham Vasculitis Activity Score version 3 in systemic vasculitis. Rheumatology (Oxford) 2011;50:899-905.

129 Exley AR, Bacon PA, Luqmani RA, et al. Development and initial validation of the Vasculitis Damage Index for the standardized clinical assessment of damage in the systemic vasculitides. Arthritis Rheum 1997;40:371-80.

130 Luqmani RA, Bacon PA, Moots RJ, et al. Birmingham Vasculitis Activity Score (BVAS) in systemic necrotizing vasculitis. QJM 1994;87:671-8.

131 de Groot K, Gross WL, Herlyn K, et al. Development and validation of a disease extent index for Wegener's granulomatosis. Clin Nephrol 2001;55:31-8.

132 Guillevin L, Lhote F, Gayraud M, et al. Prognostic factors in polyarteritis nodosa and Churg-Strauss syndrome. A prospective study in 342 patients. Medicine (Baltimore) 1996:75:17-28.

133 Merkel PA, Cuthbertson DD, Hellmich B, et al. Comparison of disease activity measures for anti-neutrophil cytoplasmic autoantibody (ANCA)-associated vasculitis. Ann Rheum Dis 2009;68:103-6.

134 Robson J, Doll H, Suppiah R, et al. Damage in the anca-associated vasculitides: long-term data from the European vasculitis study group (EUVAS) therapeutic trials. Ann Rheum Dis 2015;74:177-84

135 Alberici F, Smith RM, Jones RB, et al. Long-term follow-up of patients who received repeat-dose rituximab as maintenance therapy for ANCA-associated vasculitis. Rheumatology (Oxford) 2015;54:1153-60. 


\section{Recommendation}

136 Stassen PM, Sanders JS, Kallenberg CG, et al. Influenza vaccination does not result in an increase in relapses in patients with ANCA-associated vasculitis. Nephrol Dial Transplant 2008;23:654-8.

137 Jeffs $L S$, Peh CA, Jose MD, et al. Randomized trial investigating the safety and efficacy of influenza vaccination in patients with antineutrophil cytoplasmic antibody-associated vasculitis. Nephrology (Carlton) 2015;20:343-51.

138 Holvast A, Stegeman CA, Benne CA, et al. Wegener's granulomatosis patients show an adequate antibody response to influenza vaccination. Ann Rheum Dis 2009;68:873-8.

139 van Assen S, Agmon-Levin N, Elkayam 0, et al. EULAR recommendations for vaccination in adult patients with autoimmune inflammatory rheumatic diseases. Ann Rheum Dis.2011;70:414-22.

140 Suppiah R, Judge A, Batra R, et al. A model to predict cardiovascular events in patients with newly diagnosed Wegener's granulomatosis and microscopic polyangiitis. Arthritis Care Res (Hoboken) 2011;63:588-96.

141 Herlyn K, Gross WL, Reinhold-Keller E. [Longitudinal effects of structured patient education programs for vasculitis patients]. Z Rheumatol 2008;67: 206-10.

142 Mooney J, Spalding N, Poland F, et al. The informational needs of patients with ANCA-associated vasculitis-development of an informational needs questionnaire. Rheumatology (Oxford) 2014;53:1414-21.
143 Herlyn K, Hellmich B, Seo P, et al. Patient-reported outcome assessment in vasculitis May provide important data and a unique perspective. Arthritis Care Res (Hoboken) 2010;62:1639-45.

144 Langford CA. Update on Wegener granulomatosis. Cleve Clin J Med 2005;72:689-90, 693-7.

145 Seo P, Min YI, Holbrook JT, et al. Damage caused by Wegener's granulomatosis and its treatment: prospective data from the Wegener's Granulomatosis Etanercept Trial (WGET). Arthritis Rheum 2005;52:2168-78.

146 Basu N, Jones GT, Fluck N, et al. Fatigue: a principal contributor to impaired quality of life in ANCA-associated vasculitis. Rheumatology (Oxford) 2010;49:1383-90.

147 Basu N, McClean A, Harper L, et al. Explaining fatigue in ANCA-associated vasculitis. Rheumatology (Oxford) 2013;52:1680-5.

148 Little MA, Nightingale P, Verburgh CA, et al. Early mortality in systemic vasculitis: relative contribution of adverse events and active vasculitis. Ann Rheum Dis 2010;69:1036-43.

149 Basu N, McClean A, Harper L, et al. Markers for work disability in anti-neutrophil cytoplasmic antibody-associated vasculitis. Rheumatology (Oxford) 2014;53:953-6.

150 Walsh M, Mukhtyar C, Mahr A, et al. Health-related quality of life in patients with newly diagnosed antineutrophil cytoplasmic antibody-associated vasculitis. Arthritis Care Res (Hoboken) 2011;63:1055-61.

151 Basu N, McClean A, Harper L, et al. The characterisation and determinants of quality of life in ANCA associated vasculitis. Ann Rheum Dis 2014;73:207-11. 


\section{Erratum: Eular/ERA-EDTA recommendations for the management of ANCA-associated vasculitis}

Yates M, Watts RA, Bajema IM, et al. Eular/ERA-EDTA recommendations for the management of ANCA-associated vasculitis. Ann Rheum Dis 2016;75:1583-94. Under statement 3 the correct cyclophosphamide dosage should be $\left(0.6 \mathrm{~g} / \mathrm{m}^{2}\right)$.

Ann Rheum Dis 2017:76:1480. doi:10.1136/annrheumdis-2016-209133corr1 
Correction: EULAR/ERA-EDTA recommendations for the management of ANCA-associated vasculitis

Yates M, Watts RA, Bajema IM, et al. EULAR/ERA-EDTA recommendations for the management of ANCA-associated vasculitis. Ann Rheum Dis 2016;75:1583-94.doi:10.1136/ annrheumdis-2016-209133

Under statement 7 the correct methotrexate dosage should be (20-25 mg/week).

(C) Author(s) (or their employer(s)) 2022. No commercial re-use. See rights and permissions. Published by BMJ.

Ann Rheum Dis 2022;81:e109. doi:10.1136/annrheumdis-2016-209133corr2

(A) Check for updates 